N 6912777

\title{
GASE FILE
} NASA CR 97887 HIG-68-10

\section{ON THE EQUILIBRIUM FIGURE OF THE EARTH}

\author{
By. \\ MOHAMMAD ASADULLAH KHAN
}

SEPTEMBER 1968

Prepored for

NATIONAL ARRONAUTICS AND SPACE ADMIISTRATION UNDER GRANT NO. NGR 12.001\%045

\section{HAWAII INSTITUTE OF GEOPHYSICS UNIVERSITY OF HAWAII 
ON THE EQUILIBRIUM FIGURE OF THE EARTH

\section{By}

Mohammad Asadu1lah Khan

September 1968

Prepared for

National Aeronautics and Space Administration Grant No. NGR-12-001-045

Approved by Director

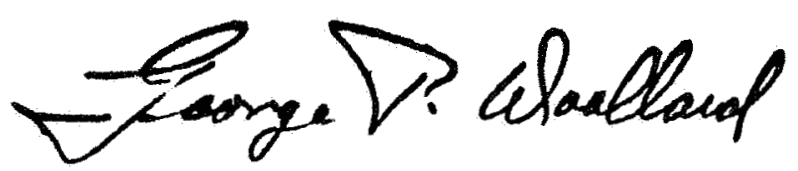

Date: 18 November 1968 



\section{CONTENTS}

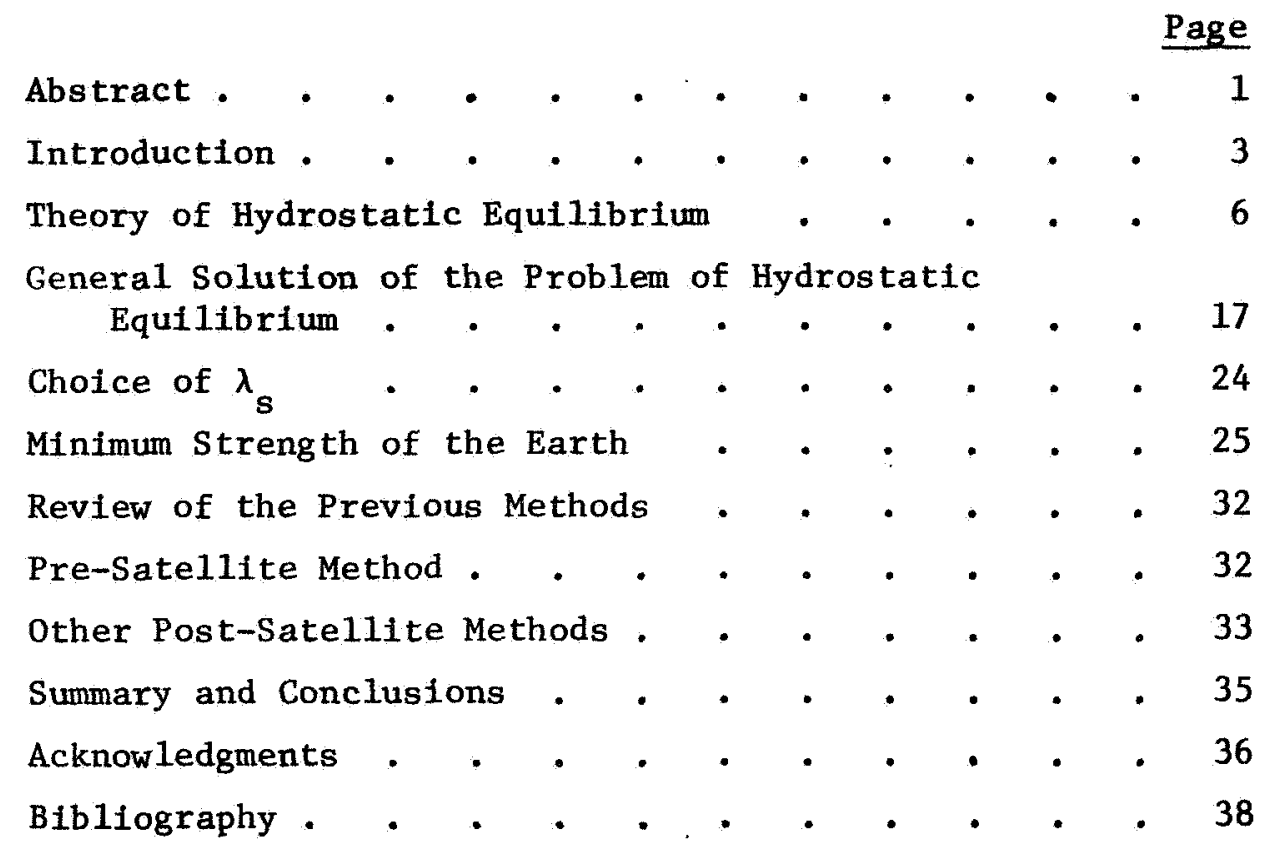




\section{ABSTRACT}

If de Sitter's hydrostatic equations are developed independent of the external potential theory, the hydrostatic geopotential coefficient $J_{h}$ occurs explicitly on the right-hand side of those equations. Since $J_{h}$ here has to be treated as on unknown in the solution, it becomes rather difficult to solve the equations independently, regardless of which of the dynamical parameters associated with the earth is taken as the initial datum. Solution is possible, however, with the help of a boundary condition derived from the extermal potential theory which neither assumes nor discounts the presence of equilibrium conditions in the earth's interior. If a general solution is constructed on this basis, the three particular solutions, usually quoted in literature, stem from it in the wake of the oppropriate assumptions. Of course, the only meaningful solution--of these-- is that corresponding to the polar moment of inertia as the initial datum. It is essential that the solution be constructed in this way in order to demonstrate clearly the correct structure of the problem of hydrostatic equilibrium.

The anomalous gravity field of the earth referred to the hydrostatic figure is compared with that referred to the intemational reference ellipsoid. 



\section{Introduction}

The hydrostatic theory of the earth, in its present form, was originally developed by Clairaut (1743). Radau (1885) simplified It by making an important substitution in Clairaut's differential equation. Originally it was hoped that a knowledge of flattening $f$, dynamical flattening $\mathrm{H}$, and $\mathrm{m}$ (defined later) would lead to some useful information about the distribution of density within the earth. The application of the theory, however, showed that with $\mathrm{m}$ and $\mathrm{H}$ fixed, widely different laws of density led to almost the same value of flattening (Jeffreys, 1962, page 152). This led to an important result: if one could assume that this theory was applicable to the real earth, i.e., if the earth was in hydrostatic equilibrium, it would be possible to compute its flattening. This would require a knowledge of the parameters $m$ and $H$ and of the distribution of density, to compute the geopotential coefficient J. The parameters $\mathrm{m}$ and $\mathrm{H}$ were quite we11-determined. The distribution of density was not very we1l-known, but this theory had indicated that the use of a reasonable density model was adequate for the computations. An obvious inference was that the value of flattening, obtained on the basis of this theory for a reasonable density distribution, would give a good approximation to the actual flattening of the earth spheroid. Such an inference was particularly welcome at that time because a precise determination of the geopotential coefficient $J$ was not possible to provide the value of flattening directly. Other methods of determining the flattening of the earth were dependent upon gravimetric or arc measurement data and hence had their obvious limitations. Doubts as to the validity of the above assumption arose, however, when Tisserand (1891) and Poincare (1910) derived $f_{h}^{-1}=297.3$ ( $f_{h}$ denotes hydrostatic flattening and $f$ real flattening) on the basis of hydrostatic theory, in contrast to the then-accepted value of $\mathrm{f}^{-1}=293.5$ which A.R. Clark had obtained from arc measurements in 1880 (Jeffreys, 1962, p. 152). Later, Hayford (1909, 1910) and Helmert (1911), using more extensive data, obtained $\mathrm{f}^{-1}=297.0$, which was quite consistent with the value obtained from hydrostatic theory within the limits of accuracy. This reaffirmed the then-prevalent opinion that the method of 
hydrostatic theory for computing the flattening of the earth was more accurate than any other available at that time (de Sitter, 1924, 1938; Jeffreys, 1948, 1962; Jones, 1954). This interesting conclusion was based on the belfef that since hydrostatic equilibrium probably existed throughout the earth's interior except for the crust and perhaps the upper mantle, the assumption of hydrostatic equilibrium for the whole earth was not unjustified. This belief persisted until artificial satellites made possible the direct determination of the geopotential coefficient $J$, from which the flattening $f$ of the best-fitting spherold could be accurately computed using the external potentlal theory alone, without recourse to either gravimetric data or geodetic arc measurement data or to the hydrostatic equilibrium theory. The currently accepted value of $f$ computed in this way is $1 / 298.25$. Since the real flattening and the hydrostatic flattening could now be computed independent of each other, any discrepancy between the real flattening $f$ and the hydrostatic flattening $f_{h}$ could now be studied, and the validity of the claim made in the pre-satelite times that $f=f_{h}$ could now be proved or disproved definitely. Henriksen (1960) used de Sitter's equations and $\mathrm{J}=1622.4 \times 10^{-6}$ to find a value of $1 / 300$ for hydrostatc flattening. Jeffreys (1963), using $\mathrm{J}=1624.17 \times 10^{-6}$ as obtained by King-Hele, Cook and Rees (1963), found a value of $1 / 299.67 \pm 0.05$ for the hydrostatic flattening, confirming the discrepancy between $f$ and $f_{h}$ which Henriksen (1960) and $0^{\prime}$ Keefe (1960) had inftially pointed out. Using the improved value of the constant of precession (Rabe, 1950), of moon-earth mass ratio $(1 / 81.303)$ and $J=1623.969 \times 10^{-6}$ (Koza1, 1964), I (Khan, 1967) obtained $1 / 299.86 \pm 0.05$ for the hydrostatic flattening by employing Jeffreys' (1963) approach, which has both the advantages of speed and simplicity. In his method, Jeffreys (1963) has carried the analytical development to first order and then applied the corrections for the second-order terms by the numerical integration of the appropriate expressions. Although Jeffreys' paper outlines the structure of the hydrostatic problem clearly, he does not use de Sitter's equations. The re has been considerable confusion regarding the computation of hydrostatic flattening from de Sitter's hydrostatic equations which 
carry the development of the theory to the second order analytically. The general belief has been that the hydrostatic flattening is and should be computed from the hydrostatic part of the equations alone, to the complete exclusion of the external potentlal theory. In order to examine the validity of this belief I re-evaluated (Khan, 1968a) the hydrostatic theory and made some modifications in de Sitter's (1924) equations in order to reinstate the geopotential coefficient $\mathrm{J}$ which de Sitter had eliminated from the right-hand side of his equations by means of a relation derived from the external potential theory. This step is essential in order to demonstrate the correct structure of the problem. When de sitter worked out his equations, the geopotential coefficient $J$ was not known accurately and he could presume that the re was no significant difference between the value of $J$ for the real earth and the value of hydrostatic $\mathrm{J}$. This assumption has been proven as incorrect ever since. However, as will be discussed later, there is theoretical justification for the elimination of $\mathrm{J}$--but the reason that we ought to avold it at this stage is that it is so well-concealed in the development of de Sitter's equations that it tends to obscure the correct structure of solution of the problem of hydrostatic equilibrium.

In this report, I first give the derivation of the modified equations followed by a description of the general solution. I then present a review of previous methods, both pre-satellite and postsate11ite.

I wish to emphasize here that the discussion given in this report pertains primarily to de Sitter's development of the hydrostatic theory. The elimination of the geopotential coefficient $J$ from the right-hand side of de Sitter's equations is certainly valid if the correct reason for it is outlined clearly. However, the prevaling situation is that it has given rise to the bellef that the hydrostatic flattening can be computed merely from the hydrostatic part of de S1tter's development of the theory without any use whatsoever of the external potential theory even if the only known datum used is the polar moment of inertia of the real earth. The principal purpose of this report is to show that if de Sitter's development is used for computing hydrostatic flattening merely from a knowledge of the earth's polar or mean moment of inertia 
(making no additional use of the satellite-determined $J$ except in the computation of the polar moment of inertia), some control from the external potential theory must be introduced.

\section{Theory of Hydrostatic Equilibrium}

Development of Modified Hydrostatic Equations:

The customary manner of representing the outer potential of a reference figure, symmetrical with reference to both an equatorial plane and the polar axis (to the order of accuracy required here) is

$$
\begin{aligned}
V= & \frac{G M}{r}\left[1-J_{2}\left(\frac{a}{r}\right)^{2} P_{2}(\sin \phi)-J_{4}\left(\frac{a_{e}}{r}\right)^{4} P_{4}(\sin \phi)\right] \\
& +\frac{1}{2} \omega^{2} r^{2} \cos ^{2} \phi
\end{aligned}
$$

where

$$
\begin{aligned}
& G=\text { gravitational constant } \\
& M=\text { mass of the earth } \\
& a_{e}=\text { equatorial radius of the earth } \\
& \omega=\text { rate of rotation of the earth } \\
& P_{2}(\sin \phi) \text { and } P_{4}(\text { sin } \phi)=\text { Legendre's polynomials } \\
& J_{2} \text { and } J_{4}=\text { zonal harmonic coefficients in the spherical } \\
& \text { harmonic representation of earth's potential }
\end{aligned}
$$

The radius vector of an ellipsoid of revolution can be represented (to the order of accuracy required here) as

$$
r=a_{e}\left(\alpha_{0}+\alpha_{2} P_{2}+\alpha_{4} P_{4}\right)
$$


whe re

$$
\begin{aligned}
& \alpha_{0}=1-\frac{1}{3} \mathrm{f}-\frac{1}{5} \mathrm{f}^{2} \\
& \alpha_{2}=-\frac{2}{3} \mathrm{f}-\frac{1}{7} \mathrm{f}^{2} \\
& \alpha_{4}=\frac{12}{35} \mathrm{f}^{2}
\end{aligned}
$$

In the foregoing expression $f$ is the flattening of the surface described by Eq. (2).

Substituting $r$ from Eqs. (2) and (3) into Eq. (1) and retaining quantities to the $O\left(f^{2}\right)$ only, we get the potential $v$ on the surface of the ellipsoid, i.e.,

$$
\begin{aligned}
V= & v_{0}\left(G M, a_{e}, m, f, J_{2}\right)+\beta_{2}\left(G M, a_{e}, m, f, J_{2}\right) \\
& +\beta_{4}\left(G M, a_{e}, m, f, J_{2}, J_{4}\right)
\end{aligned}
$$

If the ellipsoldal surface is an equipotential surface, $V$ must be constant on $1 t$, and the coefficients $\beta_{2}$ and $\beta_{4}$ must both be zero, which, among other relations, gives us the following equation

$$
\mathrm{J}_{2}=\frac{2}{3} \mathrm{f}-\frac{1}{3} \mathrm{f}^{2}+\frac{2}{21} \mathrm{mf}-\frac{1}{3} \mathrm{~m}
$$

In the derivation of Eq. (4), no assumption is made regarding the conditions existing in the earth's interior. Hence the equation is valid whether or not hydrostatic equilibrium exists in the earth's interior. Eq. (4) gives

$$
\mathrm{f}=\frac{3}{2} \mathrm{~J}_{2}+\frac{1}{2} \mathrm{~m}+\frac{15}{28} \mathrm{~J}_{2} \mathrm{~m}+\frac{9}{8} \mathrm{~J}_{2}^{2}+\frac{3}{56} \mathrm{~m}^{2}
$$


Equations (4) and (5) should relate the observed $J$ with the real flattening in a non-hydrostatic case and the hydrostatic $J$ with the hydrostatic flattening for a hydrostatic case. Hence Eq. (4) or (5) will be of the form

$$
G(J, f)=0
$$

for the non-hydrostatic case and

$$
G\left(J_{h}, f_{h}\right)=0
$$

for the hydrostatic case.

Note that $J_{h}$ and $f_{h}$ denote the hydrostatic $J$ and the hydrostatic flattening, respectively.

The real flattening of the ellipsoidal surface which best fits the geoldis given by Eq. (5) in the form of Eq. (5a), if J for the real earth is known accurately as, for example, from the regression of node of an artifictal earth satellite.

The condition of hydrostatic equilibrium can be represented analytically by the differential equation

$$
\mathrm{dp}=\delta \mathrm{dV}
$$

where $V$ is the sum of gravitational and rotational potential, $p$ the pressure at any point within the body and $\delta$ its density. This implies that $\mathrm{p}$ must be a function of $\mathrm{V}$, and $\delta$ either a constant or a function of $\mathrm{V}$ also. Since the equipotential surfaces are given by $\mathrm{V}=$ constant, $\mathrm{p}$ and $\delta$ will also assume a constant value, as obvious from Eq. (3). Thus, the condition of hydrostatic equilibrium for the earth's interior is established by stipulating that the equipotential surfaces and the surfaces of equal density be coincident. If $\beta$ represents the mean 
radius of any such surface, expressed in terms of the outer surface as $a$ unit so that $B$ ranges from 0 at the center to 1 at the outer surface, then the equation of this surface becomes

$$
r=B\left[1-\frac{2}{3}\left(f^{\prime}+\frac{2}{3} f_{h}^{2}\right) P_{2}(\sin \phi)+\frac{12}{35} f_{h}^{2} P_{4}(\sin \phi)\right]
$$

whe re

$$
f^{\prime}=f_{h}-\frac{5}{42} f_{h}^{2}
$$

Since we are developing a second order theory and thus are not interested in terms $>0\left(f^{2}\right)$, we will treat $f^{\prime 2}=f_{h}{ }^{2}$ and $m f^{\prime}=m f_{h}$, etc.

The potential $V$ at any point $(r, \phi)$ within the earth is given by (de Sitter, 1924):

$$
\begin{aligned}
V= & \frac{G W}{r}\left[D\left(1+\frac{1}{2} m_{1} \sigma^{3} \cos ^{2} \phi\right)-\frac{2}{5}\left(S \sigma^{-2}+T \sigma^{3}\right) P_{2}(\sin \phi)\right. \\
& \left.+\left(\frac{12}{35} \mathrm{Po}^{-4}+\frac{32}{105} Q \sigma^{5}\right) \mathrm{P}_{4}(\sin \phi)\right]
\end{aligned}
$$

where

$$
\begin{aligned}
\sigma & =\frac{r}{B} \\
m_{1} & =\frac{\omega^{2} a_{e}^{3}}{G M}=\frac{m}{D} \\
W & =\text { volume of the surface of which } B \text { is the mean radius }
\end{aligned}
$$




$$
\begin{aligned}
& D=\frac{3}{\beta^{3}} \int_{0}^{\beta} \delta \beta^{2} d \beta \\
& S=\frac{1}{\beta^{5}} \int_{0}^{\beta} \delta \frac{d}{d \beta}\left[\beta^{5}\left(f^{\prime}+\frac{2}{7} f_{h^{2}}{ }^{2}\right] d \beta\right. \\
& T=\int_{\beta}^{1} \delta \frac{d}{d \beta}\left(f^{\prime}+\frac{16}{21} f_{h^{2}}{ }^{2} d B\right. \\
& P=\frac{1}{\beta^{7}} \int_{0}^{\beta} \delta \frac{d}{d \beta}\left[\beta^{7}\left(f_{h}{ }^{2}+\frac{8}{9} k\right)\right] d B \\
& Q=2 \int_{\beta}^{1} \delta \frac{d}{d \beta}\left(\frac{k}{\beta^{2}}\right) d B
\end{aligned}
$$

In these formulas $\delta$ is the density, expressed in terms of mean density as a unit, and $D$ is the mean density within the surface $B$, expressed in the same unit. For the outer surface

$$
\begin{aligned}
& D_{s}=1 \\
& S_{s}=\frac{5}{3} J\left(1+\frac{2}{3} f_{h}\right) \\
& P_{s}=\frac{35}{12} J_{4} \\
& T_{s}=0 \\
& Q_{s}=0
\end{aligned}
$$

The condition that the surface, described by Eq. (6), be an equipotential surface, gives as one of the relations the following:

$$
D\left(f^{\prime}+\frac{2}{7} f_{h}^{2}-\frac{1}{2} m_{1}\right)-\frac{3}{5}(S+T)=\frac{4}{21} f(m-3 T)
$$


Differentiating Eq. (9) and introducing a new varlable $n$, defined by

$$
\eta=\frac{d \log f^{\prime}}{d \log B}=\frac{\beta}{f^{\prime}}, \frac{d f^{\prime}}{d \beta}
$$

we get

$$
\eta\left[D\left(1+\frac{4}{7} f_{h}\right)-\frac{4}{21} m+\frac{4}{7} T\right]=3 D\left(1+\frac{2}{7} f_{h}\right)-\frac{3 S}{f^{\prime}}
$$

With the help of Eq. (8), the above equation gives us a boundary condition for the outer surface which can be written as

$$
\eta_{s} f^{\prime}\left(1+\frac{4}{7} f_{h}-\frac{4}{21} m\right)=3 f^{\prime}\left(1+\frac{2}{7} f_{h}\right)-5 J_{h}\left(1+\frac{2}{3} f_{h}\right)
$$

where $n_{s}$ denotes the surface value of $n$.

Again, by differentlating Eq. (9a), we can get

$$
B \frac{d \eta}{d B}+\eta^{2}+5 \eta-2 \zeta(1+n)-\frac{4}{21} \zeta \xi=0
$$

In which

$$
\zeta=-\frac{\beta}{D} \frac{d D}{d \beta}=3\left(1-\frac{\delta}{D}\right)
$$

and

$$
\xi=7 \frac{m}{D}(1+n)-3 f_{h}(1+n)^{2}-4 f
$$


Radau (1885) transformed Eq. (11) to the form

$$
\frac{d}{d B} D B^{5} \sqrt{1+\eta}=5 D B^{4} F(n)
$$

where

$$
F(n)=\frac{1+\frac{1}{2} n-\frac{1}{10} n^{2}+\frac{2}{105} \zeta \xi}{\sqrt{1+n}}
$$

$F(n)$ has the remarkable property that it lies very near unity, 1t's maximum departure being 0.0007 . If $1+\lambda$ denotes the average value of this function over the range of integration, we can write

$$
\int_{0}^{\beta} D \beta^{4} d \beta=\frac{D}{5} \frac{\sqrt{1+\eta}}{1+\lambda} \beta^{5}
$$

which, for the outer surface, assumes the form

$$
\int_{0}^{1} D B^{4} d \beta=\frac{1}{5} \frac{\sqrt{1+n_{s}}}{1+\lambda_{s}}
$$

Note that $n_{s}$ and $\lambda_{s}$ are the values of parameters $n$ and $\lambda$ for the outer surface.

Now in our units, we have

$$
\mathrm{Ma}_{e}^{2}=\frac{4 \pi}{3}\left(1+\frac{2}{3} \mathrm{f}_{\mathrm{h}}\right)
$$

The polar moment of inertia $C$ can be written as

$$
\begin{aligned}
C= & \frac{8}{15} \pi \int_{0}^{1} \delta \frac{d}{d \beta}\left[\beta^{5}\left(1+\frac{2}{3} f_{h}\right)\right] d B=\frac{8}{3} \pi \int_{0}^{1} \delta \beta^{4} d B \\
& +\frac{2}{3}(C-A)
\end{aligned}
$$


where $A$ is the moment of inertia about the equatorial diameter. With

$$
\begin{aligned}
& H=\frac{C-A}{C} \\
& J_{h}=\frac{3}{2} \frac{C-A}{M a_{e}^{2}} \\
& q=\frac{3}{2} \frac{C}{M a_{e}^{2}}
\end{aligned}
$$

and hence

$$
J_{h}=q H \text {, }
$$

we obtain another boundary condition for the outer surface in the form

$$
q=3\left(1-\frac{2}{3} f_{h}+\frac{4}{9} f_{h}^{2}\right) \int_{0}^{1} \delta \beta^{4} d \beta+\frac{2}{3} J_{h}
$$

As stated before, if $\mathrm{D}$ is the mean density within the surface $B$ expressed in terms of the mean density as a unit, we have

$$
D=\frac{3}{\beta^{3}} \int_{0}^{\beta} \delta \beta^{2} d \beta
$$

which on differentiation with respect to $B$ gives

$$
\frac{-\beta}{D} \frac{d D}{d B}=3\left(1-\frac{\delta}{D}\right)
$$

or

$$
\delta=D\left|1+\frac{1}{3} \frac{\beta}{D} \frac{d D}{d \beta}\right|
$$


Substituting this value of $\delta$ in Eq. (14) we get

$$
q=3\left(1-\frac{2}{3} \mathrm{~F}_{h}+\frac{4}{9} \mathrm{f}_{h}^{2}\right) \int_{0}^{1} \mathrm{D}\left(1+\frac{1}{3} \frac{\beta}{D} \frac{d D}{d \beta}\right) \beta^{4} d \beta+\frac{2}{3} J_{h}
$$

which, with the help of Eq. (13a) can be finally transformed into

$$
q=1-\frac{2}{3} f_{h}+\frac{2}{3} J_{h}+\frac{4}{9} f_{h}^{2}-\frac{2}{5}\left(1-\frac{2}{3} f_{h}+\frac{4}{9} f_{h}{ }^{2}\right) \frac{\sqrt{1+\eta_{s}}}{1+\lambda_{s}}
$$

Equation (10) can be simplified to the form

$$
\left.\eta_{s} f^{\prime}=3 f^{\prime}-\frac{6}{7} f_{h}^{2}+\frac{4}{7} m f_{h}-J_{h} \mid 5+\frac{10}{21} f_{h}+\frac{20}{21} m\right)
$$

From Eq. (15) one obtains

$$
n_{s}=\left(1+\left.\lambda_{s}\right|^{2}\left[\frac{\left(1-q-\frac{2}{3} f_{h}+\frac{2}{3} J_{h}+\frac{4}{9} f_{h}^{2} \mid\right.}{\frac{2}{5}\left(1-\frac{2}{3} f_{h}+\frac{4}{9} f_{h}^{2} \mid\right.}\right]^{2}-1\right.
$$

At this point, if we can assign a reasonable value to $\lambda_{s}$ (for example, $\lambda_{s}=0$ as explained later), we can substitute $\eta_{s}$ from Eq. (17) into Eq. (16) and obtain an equation in $J_{h}$ and $f_{h}$ from which a series of values of $f_{h}$ can be obtained corresponding to arbitrary values of $\mathrm{J}_{\mathrm{h}}$. However, it is convenient to get an expression which gives $f_{h}$ explicitly in terms of other parameters. This can be done by writing Eq. (17) as

$$
n_{s}=\frac{25}{4} F^{2} q^{\prime 2}\left(\frac{1-\Delta_{1}}{1-\Delta_{2}}\right)^{2}-1
$$

where 


$$
\begin{aligned}
& q^{\prime}=1-q \\
& \Delta_{1}=\frac{\frac{2}{3}\left(f_{h}-J_{h}-\frac{2}{3} f_{h}^{2}\right)}{q^{\prime}} \\
& \Delta_{2}=\frac{2}{3}\left(f_{h}-\frac{2}{3} f_{h}^{2}\right) \\
& F=1+\lambda_{s}
\end{aligned}
$$

It 18 Instructive to note that $\Delta_{1}$ and $\Delta_{2}$ are both of the order of flattening.

Simplifying Eq. (18), one obtains

$$
\begin{aligned}
n_{s}= & \frac{25}{4} F^{2} q^{\prime 2}\left[1+2\left(\Delta_{2}-\Delta_{1}\right)\right. \\
& +\left(\Delta_{1}^{2}+3 \Delta_{2}^{2}-4 \Delta_{1} \Delta_{2} \mid\right]-1=n_{0}+n_{1}+n_{2}
\end{aligned}
$$

where

$$
\begin{aligned}
& n_{0}=\frac{25}{4} F^{2} q^{\prime 2}-1 \\
& n_{1}=\frac{25}{2} F^{2} q^{\prime 2}\left(\Delta_{2}-\Delta_{1}\right)=\frac{25}{3} F^{2} q^{\prime}\left(J_{h}-q f_{h}+\frac{2}{3} q f_{h}^{2}\right)
\end{aligned}
$$

and

$$
\eta_{2}=\frac{25}{4} F^{2} q^{\prime 2}\left|\Delta_{1}{ }^{2}+3 \Delta_{2}{ }^{2}-4 \Delta_{1} \Delta_{2}\right|
$$

Note that the quantity $n_{1}$ is of the order of $f_{h}$, whereas $n_{2}$ is of the order of $f_{h}^{2}$. 
Using this value of $\eta_{s}$, Eq. (16) can be written as

$$
A f_{h}^{2}+\left(n_{0}-3+\delta_{1}\right) f_{h}+5 J_{h}+\delta_{2}=0
$$

where we have put

$$
\begin{aligned}
& A=\frac{17}{14}-\frac{5}{42} n_{0}-\frac{25}{3} F^{2} q q^{\prime} \\
& \delta_{1}=\frac{25}{3} F^{2} q^{\prime} J_{h}-\frac{4}{7} m+\frac{10}{21} J_{h}
\end{aligned}
$$

and

$$
\delta_{2}=\frac{20}{21} \mathrm{~m} \mathrm{~J}_{\mathrm{h}}
$$

Note that $\delta_{1}$ is approximately of the order of $f_{h}$, whereas $\delta_{2}$ is of the order of $\mathrm{f}_{\mathrm{h}}{ }^{2}$.

Equation (22) gives the required expression for $f_{h}$ which, correct to the second order of sma1l quantities, is

$$
\begin{aligned}
f_{h}= & \frac{1}{\left(n_{0}-3\right)}\left[-\left(5 J_{h}+\delta_{2}\right)+\frac{\left(5 J_{h}+\delta_{2}\right) \delta_{1}}{\left(n_{0}-3\right)}\right. \\
& \left.-\frac{25 A J_{h}^{2}}{\left(n_{0}-3\right)^{2}}\right]
\end{aligned}
$$

It is interesting to see that in this development, the expression for $f_{h}$ corresponding to the first order theory is

$$
f_{h}=\frac{5 J_{h}}{3-n_{0}}
$$


General Solution of the Problem of Hydrostatic Equilibrium

To solve the problem of hydrostatic equilibrium we have to examine hydrostatic Eq. (24) which can be written as

$$
F\left(m, H, J_{h}, f_{h}\right)=0
$$

In terms of the basic parameters, the above equation can be written as

$$
F\left(C, A, f_{h}, w, a, M\right)=0
$$

If the hydrostatic equilibrium exists, the figure of the earth predicted from the external potential theory should be coincident with the hydrostatic figure (See Footnote, p. 37). This gives an important boundary condition of the problem, i.e, if the hydrostatic equilibrium exists in the earth's interior, Eqs. (5) and (24) must match at the outer boundary of the earth. Hence,

$$
F\left(m, H, J_{h}, f_{h}\right)=G\left(J_{h}, f_{h}\right)
$$

or more specifically,

$$
\begin{aligned}
& \frac{1}{3-n_{0}}\left[\left(5 J_{h}+\delta_{2}\right)+\frac{\left(5 J_{h}+\delta_{2}\right) \delta_{1}}{3-n_{0}}+\frac{25 A J_{h}^{2}}{\left(3-n_{0}\right)^{2}}\right] \\
& =\frac{1}{2} J_{h}^{2}+F_{1} J_{h}+F_{2}
\end{aligned}
$$

where

$$
F_{1}=1+\frac{5}{14} m
$$




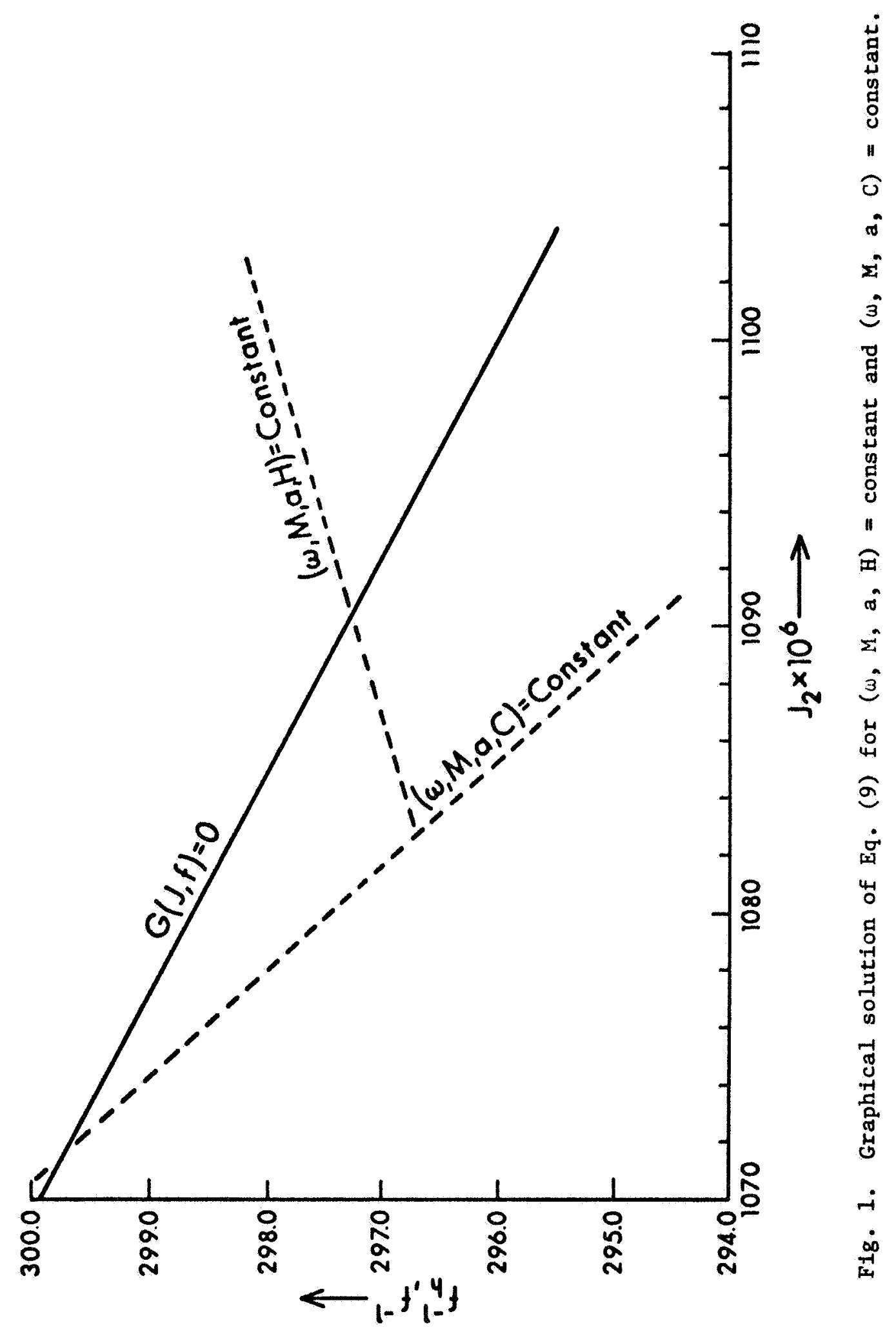




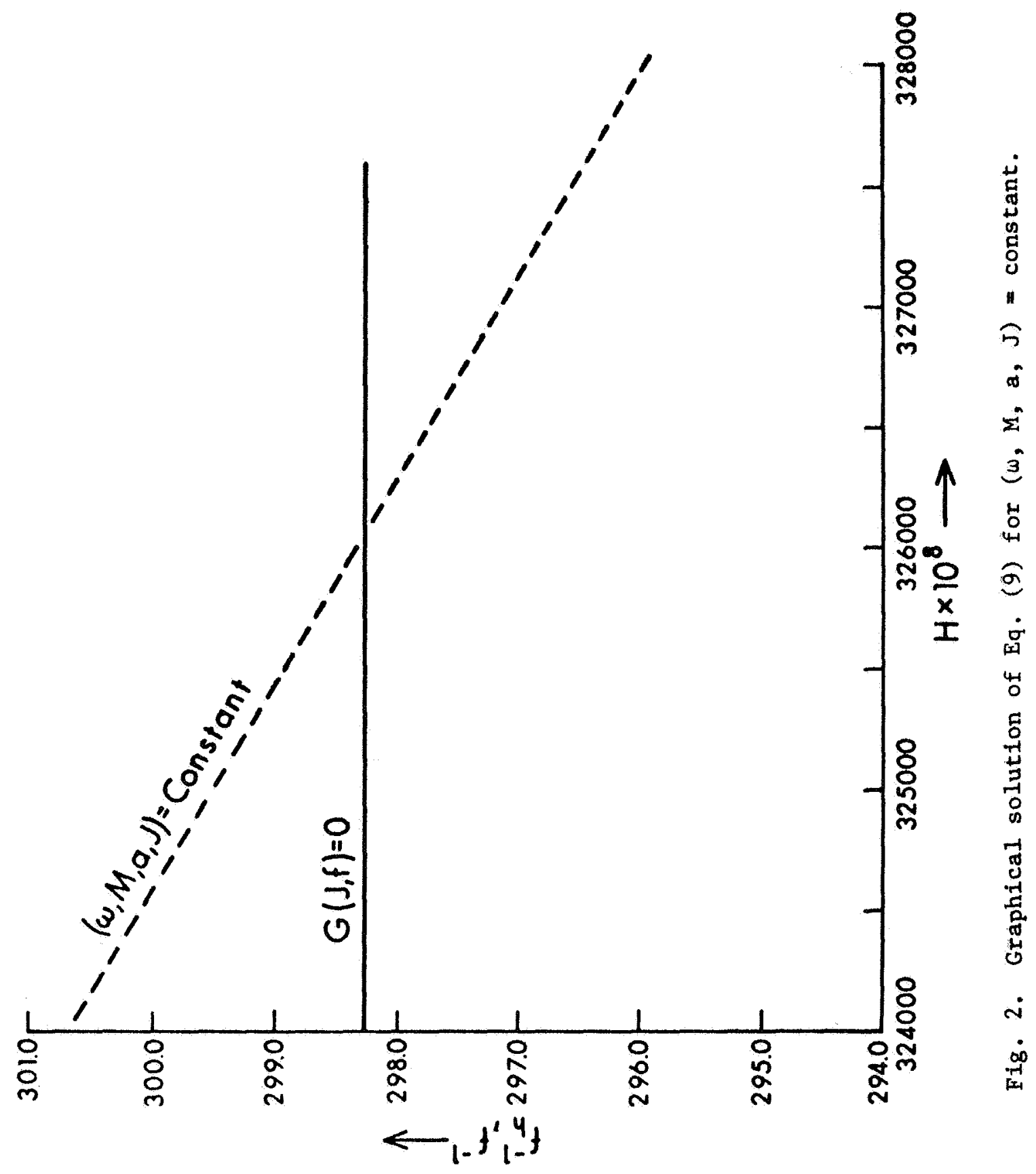




$$
F_{2}=\frac{1}{2} m+\frac{3}{56} m^{2}
$$

Other quantities on the right hand side of Eq. (24) are defined previously.

Equation (27) gives the general solution of the problem of hydrostatic equilibrium. The particular solutions are obtained by examining the left-hand side of Eq. (27) in the form of Eq. (26) or (26a) and by properly defining the basic parameters occurring in that equation. If the rate of rotation $\omega$ and the mass $M$ are chosen to be the same for the hydrostatic earth and the real earth, there are three possible solutions which correspond to the following boundary conditions

$$
\begin{aligned}
& (\omega, M, a, C)=\text { constant } \\
& (\omega, M, a, H)=\text { constant } \\
& (\omega, M, a, J)=\text { constant }
\end{aligned}
$$

Graphical solutions of the general hydrostatic Eq. (27) are given in Figure 1 for the first two cases and in Figure 2 for the third case. The plots of the function $G\left(J_{h}, f_{h}\right)$ are shown by solid curves, whereas those of $F\left(m, H, J_{h}, f_{h}\right)$ subject to the set of boundary conditions mentioned in Eq. (28) are shown by broken-line curves. It is obvious from the shape of these plots that each set has an unique intersection point in the region in which we are interested. Note that in Figure 1 the abcissa is scaled in terms of $\mathrm{J}_{2 \mathrm{~h}}$ where $\mathrm{J}_{2 \mathrm{~h}}=\frac{2}{3} \mathrm{~J}_{\mathrm{h}}$. In a11 the solutions, $\lambda_{s}$ has been taken equal to zero. It is shown later (see Fig. 3) that reasonable variations in values of $\lambda_{s}$ do not affect the solution critically. The rate of rotation $\omega$ is treated as constant via the parameter $m$ in all the solutions given in Figures 1 and 2 .

For the case when $(\omega, M, a, C)=$ constant, the hydrostatic flattening is $f_{h}^{-1}=299.75 \pm 0.05$ and the hydrostatic $J$ is $J_{h}=1607.49$ $\times 10^{-6}$. This value is about the same as reported by Henriksen (1960), 


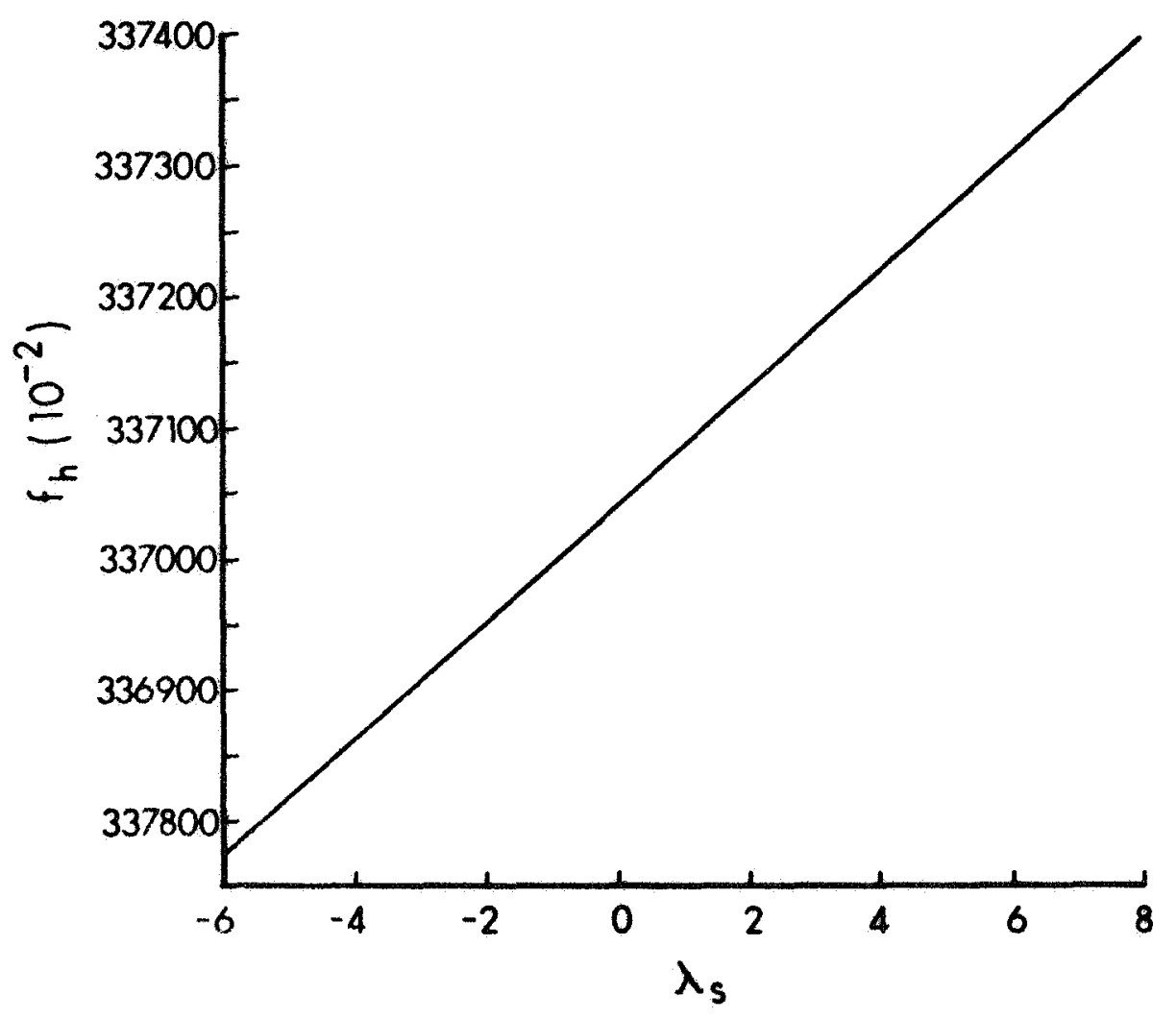

Fig. 3. Influence of $\lambda_{s}$ on the value of $f_{h}$.

O'Keefe (1960), and in a previous paper by me (Khan, 1967). However, as much as one can gather from the post-satellite literature, the method reported previous ly ( $\underline{0}$ 'Keefe in footnote to Munk \& MacDonald, 1960) certainly does not profess (Khan, 1968a, b) to take care of the boundary condition noted above. The fact that the two methods give identical numerical results can be traced to de Sitter's specific elimination of the geopotential coefficient $J$ from the hydrostatic equations in order to obtain his equations for the hydrostatic theory. This elimination remained undetected (Khan, 1968a, b) when initial results on hydrostatic flattening were reported and led to the erroneous belief that the then- 
reported value of hydrostatic flattening was obtained from a solution of the hydrostatic equation alone. The net effect of this elimination is, in fact, the same as allowing for the above-noted boundary condition. However, it should be clear now that if the above value of $f_{h}$ is preferred, the method reported in this report is the correct way to obtain it. As usually believed, for this model the hydrostatic flattening is smaller than the real flattening.

The merit of this solution lies in the fact that the polar moment of inertia of the hydrostatic earth is equal to that of the real earth, as obtained from observational data on the geopotential coefficient $J$ and the dynamical flattening $\mathrm{H}$. Consequently, it is possible to avoid certain dynamical complications (mentioned later) which arise when the moment of inertia of the hydrostatic model is taken different from that of the real earth.

For the case when $(\omega, M, a, H)=$ constant, the hydrostatic flattening is $f_{h}=297.29 \pm 0.05$ and the corresponding hydrostatic $J$ is $J_{h}=1623.225$ $\mathrm{x} 10^{-6}$. This value of hydrostatic flattening is very near the flattening obtained in pre-satellite times. The pre-satellite method essentially consisted of predicting $\mathrm{J}$ from the hydrostatic theory and using it in Eq. (5) to compute hydrostatic flattening which was then assumed to give the best approximation to the real flattening. Thus, the pre-satellite method will tend to give similar results as the solution proposed here, if the only data used in the solution is the dynamical flattening $\mathrm{H}$. Note that the precise determination of the geopotential coefficient $J$ has not changed the method of solution. It has merely made possible the determination of the earth's moment of inertia which provides a better initial datum than the dynamical flat tening $\mathrm{H}$.

However, the polar moment of inertia of this hydrostatic model is greater than the real earth--and this introduces some dynamical complications. It would imply a change in the radial stratification of the earth, such as would result because of the equatorial bulge of the real earth being more compressed and consequently the real earth having a higher density gradient than the hydrostatic state would require. This creates the problem of suggesting some reasonable physical phenomenon responsible for such a process. Some increase in the polar 
moment of inertia could possibly be accounted for by the fact that when the earth readjusts to the equilibrium shape defined by the above mode1, there will be an increase in the polar moment of inertia because of the expansion of the equatorial bulge to conform to the new figure. Approximate calculations show, however, that this factor can account only for a sma11 fraction of the total variation required by this model. This is the hydrostatic model of pre-satellite times when the geopotential coefficient $J$ for the real earth was not precisely known and hence, the moment of inertia of the real earth could not be determined. For the hydrostatic model $(\omega, M, a, J)=$ constant, the solution of the equation $G\left(J_{h}, f_{h}\right)=0$ will obviously give a constant value of flattening, as can be seen from Figure 2. The hydrostatic flattening for this model is $f_{h}^{-1}=298.29 \pm 0.05$ and the corresponding hydrostatic value of $\mathrm{H}$ is $\mathrm{H}=3260.50 \times 10^{-6}$. However, the polar moment of inertia of the hydrostatic model is greater than that of the real earth. The dynamical problems encountered in this case are again of a similar nature to those enumerated for the second hydrostatic model.

The results of the above three solutions are summarized in Table 1 .

TABLE 1. Possible Hydrostatic Figures Obtained from the General Solution $\underline{a}$, and Data for the Real Earth $\underline{b}$

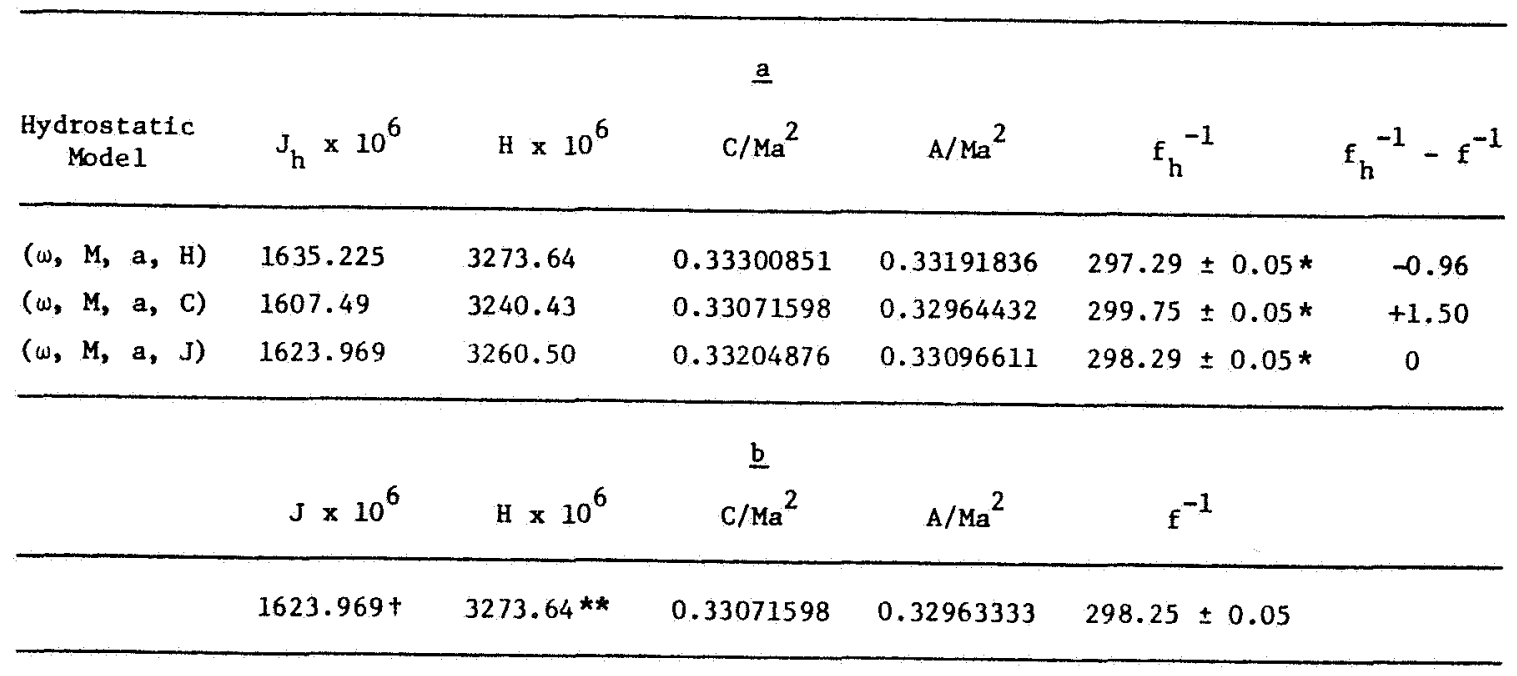

* Based on $m=0.00344980$ (Khan, 1967; Jeffreys, 1964)

+ Rozat (1964)

** Khan (1967) 


\section{Choice of $\lambda_{S}$}

In the above discussion the value of $\lambda_{S}$ is taken as zero. As pointed out earlier, the function $F(\eta)$ always lies very near unity, its maximum deviation being of the order of $10^{-4} \cdot \lambda_{\mathrm{s}}$ which is the value of $\lambda$ for the outer surface, is given by Bullard (1948) as

$$
\lambda_{s}=(1.6 \pm 1.8) 10^{-4}
$$

This estimate, however, is based on the density distribution suggested by Bullen $(1940,1942)$. Jeffreys (1963), using a simplified density model, finds $\lambda_{\mathrm{s}}=1.3 \times 10^{-4}$ and points out that if $\lambda_{\mathrm{s}}=0$ instead, the resulting $\mathrm{f}_{\mathrm{h}}$ is greater by $6 \times 10^{-7}$ only. Khan (1968a) showed that slight variations in the value of $\lambda_{s}$ do not affect the value of $f_{h}$ to any great extent, as is apparent from Figure 3. It seems legitimate, therefore, to take $\lambda_{s}=0$ for initial calculations. Note that Henriksen (1960) also took $\lambda_{s}=0$.

Table 2 summarizes some of the more important values of $\lambda_{s}$ and $n_{s}$ as obtained by different investigators.

TABLE 2. Comparison of Some Hydrostatic Theory Parameters

\begin{tabular}{llll}
\hline & & $\lambda_{s}$ & $n_{s}$ \\
\hline de Sitter & $(1924)$ & $(4.4 \pm 1.5) 10^{-4}$ & 0.5589 \\
Bu11ard & $(1948)$ & $(1.6 \pm 1.8) 10^{-4}$ & 0.565 \\
Jeffreys & $(1963)$ & $1.3 \times 10^{-4}$ & 0.5587 \\
Khan & $(1967)$ & $-\ldots .5869$ & 0. \\
\hline
\end{tabular}


Minimum Strength of the Earth

The stress differences arising because of the departure of the earth from hydrostatic equilibrium are given by Jeffreys (1943, 1963). On the supposition that the stresses are supported by strength (1) down to the core, or (2) down to a depth of 0.1 of the earth $s$ radius, the strength $\mathrm{S}$ needed to support the $\mathrm{P}_{2}$ inequality is given as follows:

For the hydrostatic mode1 ( $\left.\omega_{3} M, a, C\right)=$ constant:

$$
\begin{aligned}
& \text { Case } 1 \\
& \quad \mathrm{~S}=4.3 \times \Delta \mathrm{J}_{2} \times 10^{12} \text { dynes } / \mathrm{cm}^{2}=4.7 \times 10^{7} \text { dynes } / \mathrm{cm}^{2} \\
& \underline{\text { Case } 2} \\
& \quad \mathrm{~S}=7.9 \times \Delta \mathrm{J}_{2} \times 10^{12} \text { dynes } / \mathrm{cm}^{2}=8.7 \times 10^{7} \text { dynes } / \mathrm{cm}^{2}
\end{aligned}
$$

\section{Gravity Field Referred to the Equilibrium Figure}

The first hydrostatic model (obcained from the earth's polar moment of inertia as the datum) is used as reference for computing the anomalous gravity field of the earth from Kozai's (1964) zonal anc Gaposhkin's (1966) tesseral harmonic coefficients. If the anomalous gravity field is to be used for any studies regarding the earth's crust and mantle, it must be computed with reference to the equilibrium figure ('Keefe and Kaula, 1963; 每Keefe, 1965; Fischer, 1967) of the earth, because such a figure is a figure of zero stress, and departures from it will, inter alia, be indicative of the hydrostatic stresses existing in the earth's crust and mantle. Figure 4 shows the gravity anomalies referred to an ellipsoid with flattening 1/299.75. The gravity anomalies referred to the intemational reference ellipsoid are shown in Figure 5. It is obvious from a comparison of Figure 4 with Figure 5 how the picture of the anomalous gravity field is a function of the model adopted as the reference. This is also evident

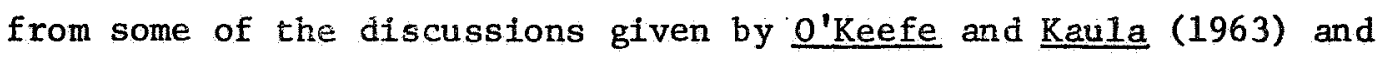
Fischer (1967). In any case it is clear that the satellite-determined 


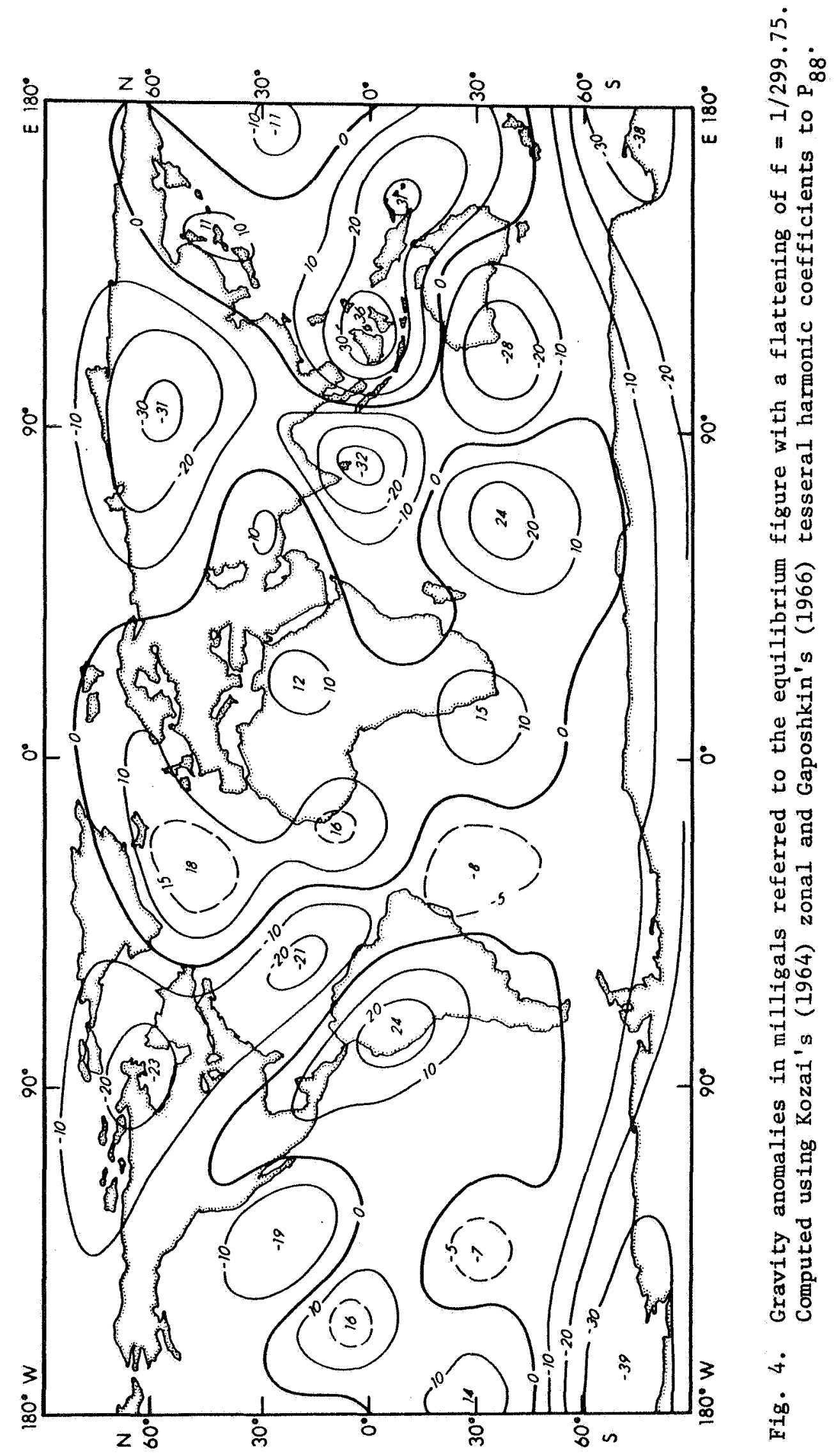




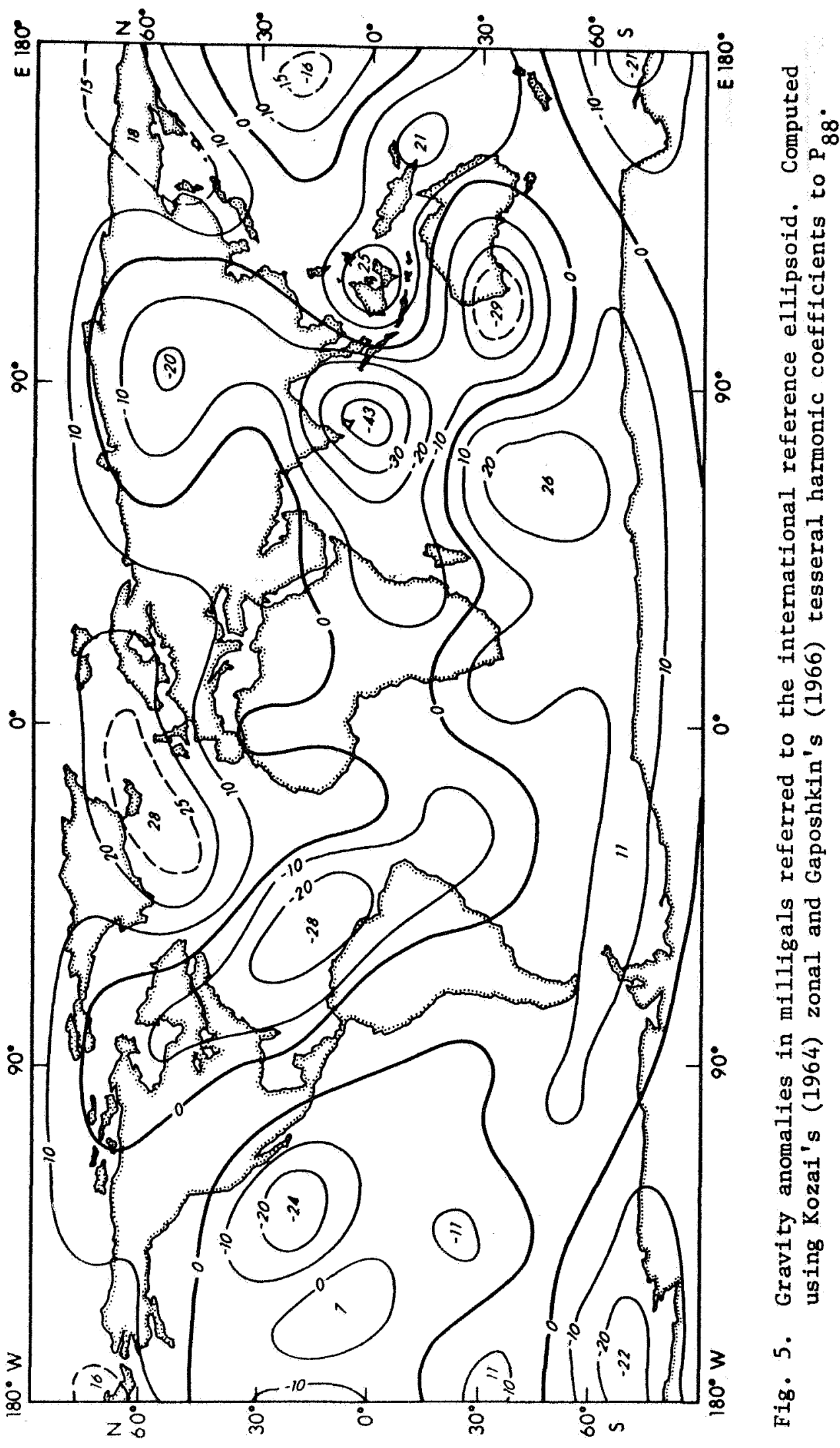




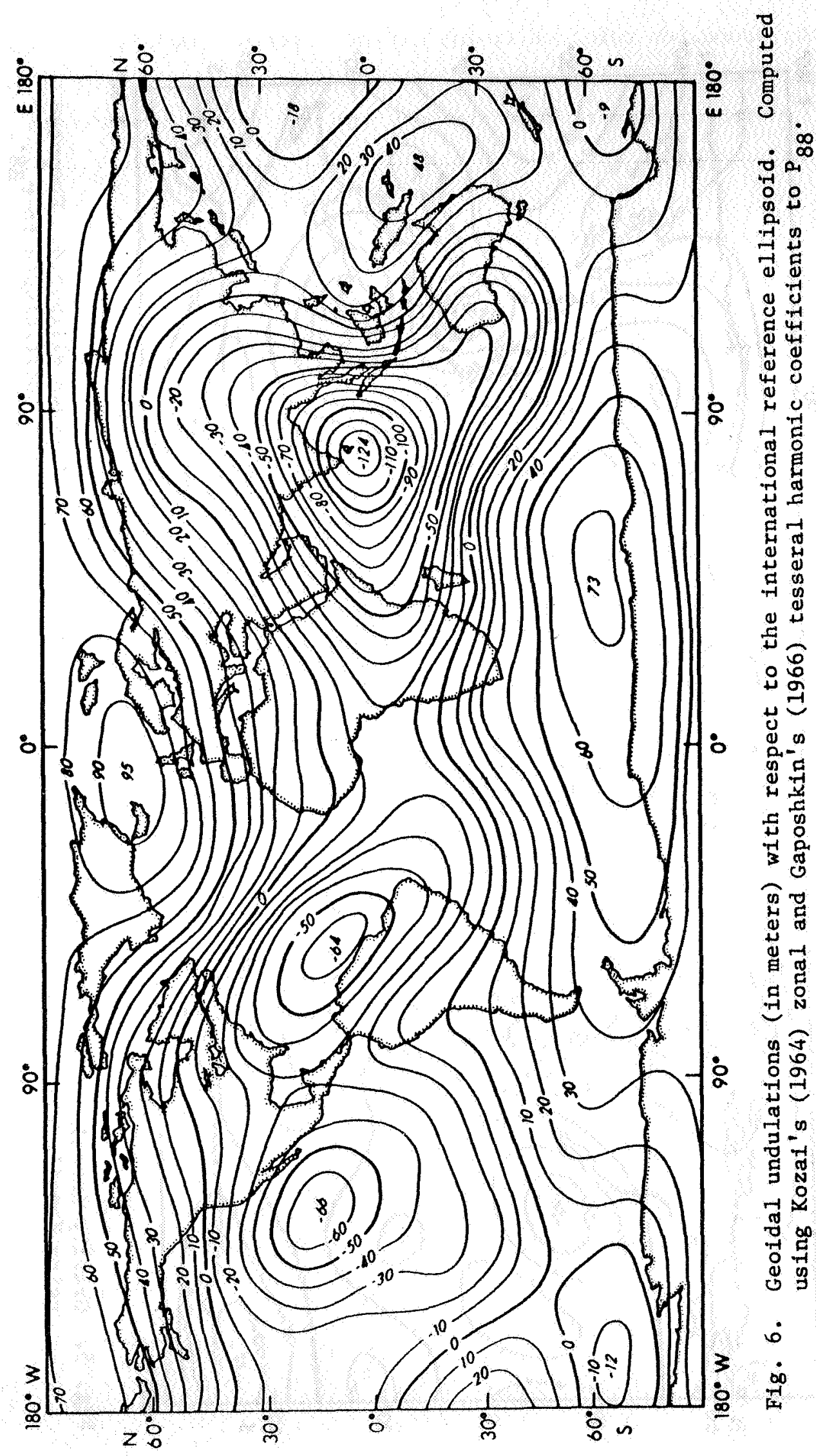


gravity anomalies referred to any of the reference models do not exceed about $43 \mathrm{milligals}$ for an 8 th harmonic representation of the gravity field, as seen from Figures 4 and 5 . The most pronounced gravity anomaly in Figure 5 is negative and occurs in the Indian Ocean just to the south of Ceylon. The magnitude of this anomaly reduces by about $11 \mathrm{mi} 11$ igals to about $32 \mathrm{milligals}$ (Fig. 4) when the equilibrium figure is used as the reference. However, in that case the positive gravity anomaly over the islands of New Guinea and Borneo becomes accentuated by an almost equal amount and becomes the most pronounced feature. Also the negative gravity anomaly located to the east of Zapadno and Sibirskaya in USSR becomes more pronounced by about 11 milligals. The we11-pronounced positive anomaly to the southsouthwest of Iceland in Figure 5 is less pronounced in Figure 4. The two negative anomalies flanking the southern tip of North America are equally observable in both the representations, while the positive anomaly over and around Peru is much more pronounced in Figure 4. The negative anomaly over the Hudson Bay area is decidedly more pronounced in Figure 4. Several other contrasting features of interest can be pointed out from a study of the two gravity field representations. It is interesting to compare the variance of the anomalous gravity field with respect to the various reference figures:

Variance of the anomalous gravity field with respect to the equilibrium figure $=145 \mathrm{mgal}^{2}$.

Variance of the anomalous gravity field with respect to the international reference ellipsoid = $136 \mathrm{mgal}^{2}$.

Figures 6 and 7 show the geoidal undulations with respect to the international reference ellipsoid and the equilibrium figure, respectively. Figure 8 is the astrogeoid computed with reference to a flattening of $1 / 299.67$ and is taken from Fischer (1967). A comparison of Figure 7 with Figure 8 shows some very interesting features. The geoid depression at latitude $60^{\circ}$ north of Tomsk in USSR is colncident in location in Figures 7 and 8 but it does not seem to match very closely in magnitude. However, there is no astrogeodetic data farther north of latitude $60^{\circ}$ in the location of this particular depression and it is difficult to say whether or not this depression will deepen farther 


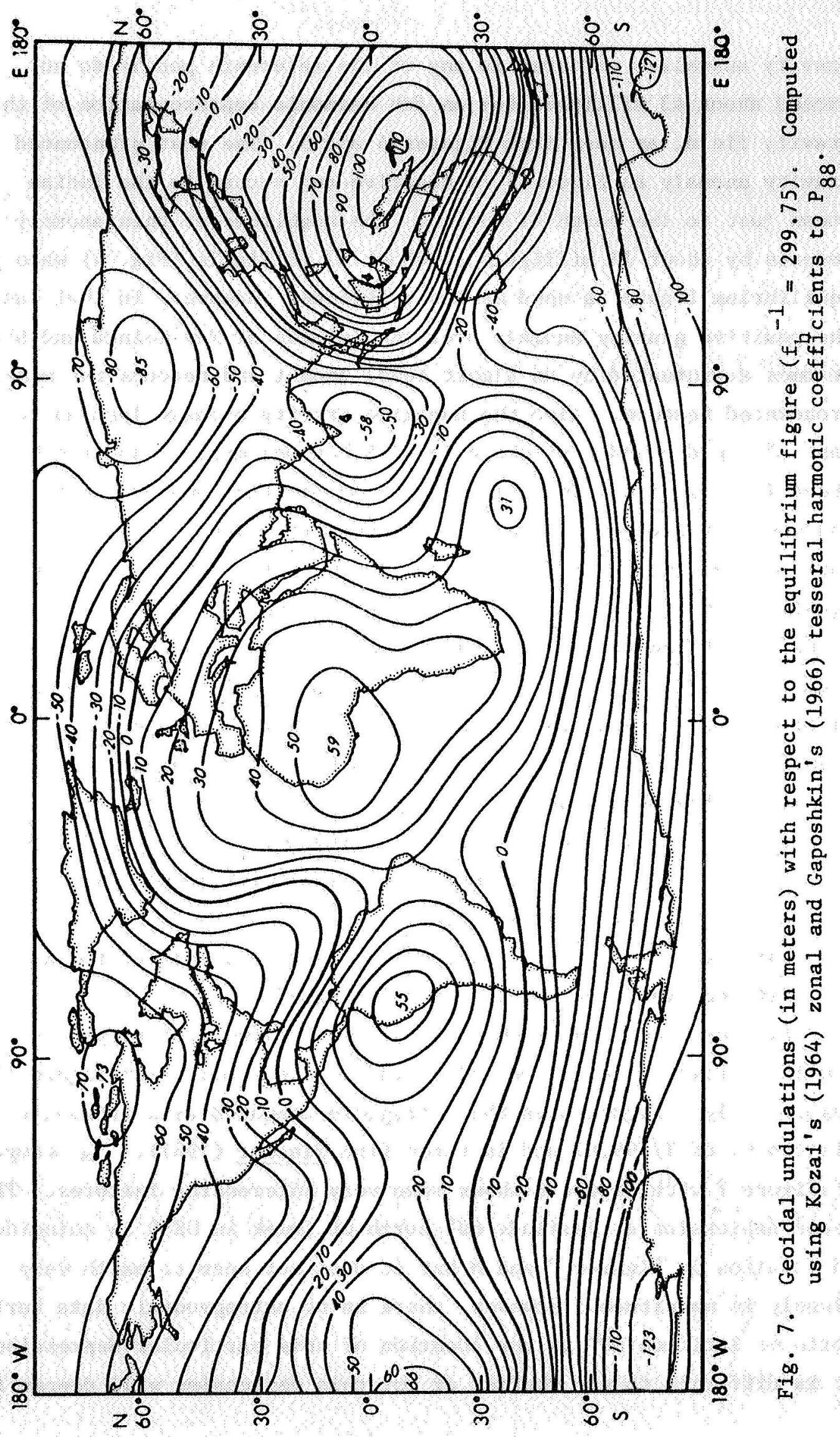




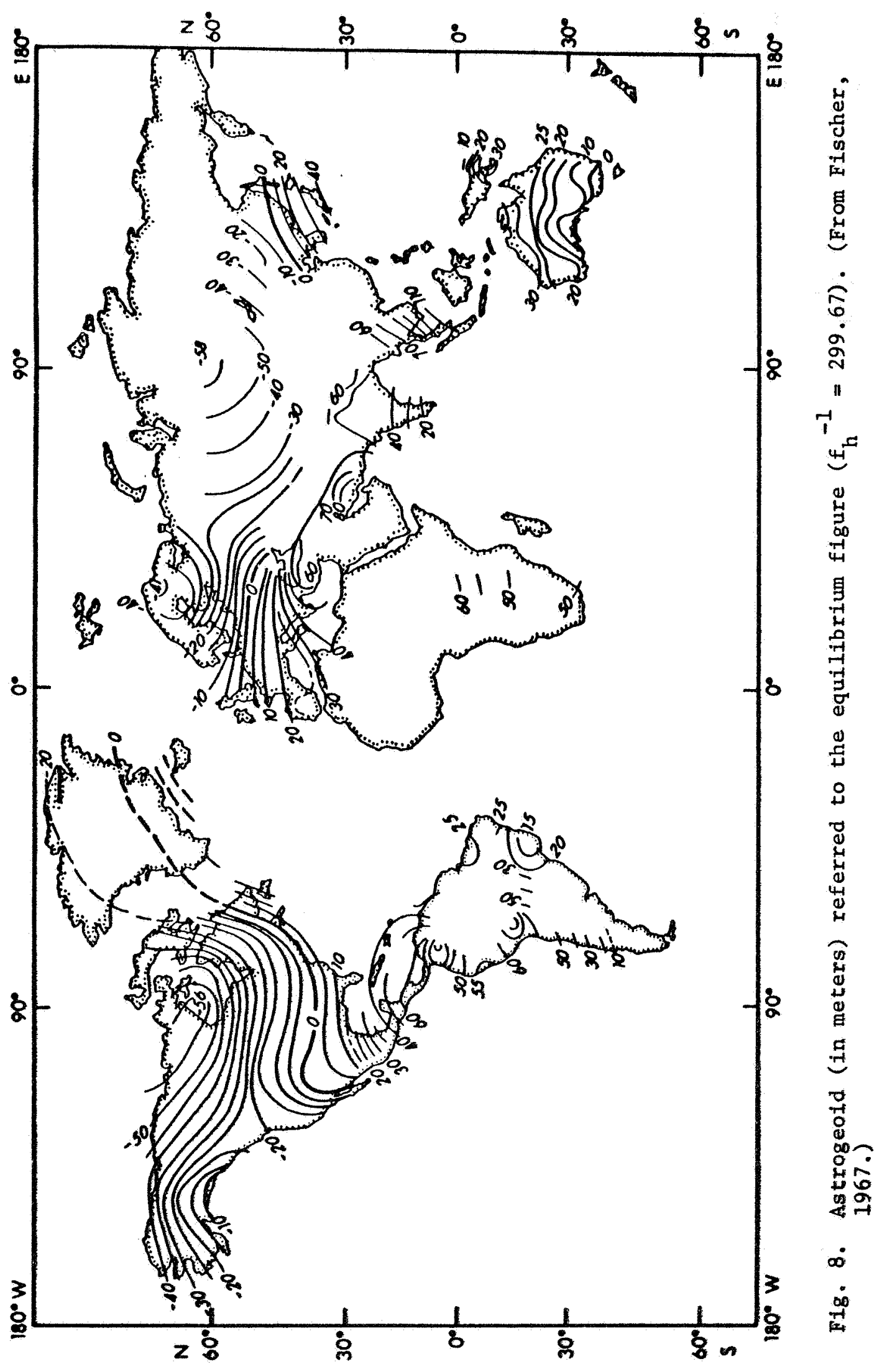


north. The geoidal low over Huds on Bay is equally well represented in both figures. A comparison of the geoidal high over the northwest and west coast of South America in Figure 7 with its counterpart in Figure 8 is strikingly noteworthy. Over Africa the two geoidal representations do not agree too we1l, but it should be noted that astrogeodetic data for the continent of Africa are very sparse. The two we11-pronounced features of the satellite geoid, i.e., the geoidal low in the Indian Ocean and the geoidal high over the Solomon Islands area, cannot be compared because practically no astrogeodetic data are available for those areas. The agreement in the major features of the two geoidal representations computed from two independent sources is, among other things, indicative of the appropriateness of the figure of reference to which the satellite and the astrogeodetic geoid are referred.

\section{Review of the Previous Methods}

Below we give a very brief review of the previous methods, examining them in perspective in the light of the general solution.

Pre-Satellite method:

If we eliminate $J_{h}$ from the right-hand side of Eq. (15) and (16) with the help of Eq. (4), we obtain

$$
\begin{aligned}
& q=1-\frac{1}{3} m+\frac{1}{9} f_{h}^{2}+\frac{2}{21} m f_{h}=\frac{2}{5}\left(1-\frac{2}{3} f_{h}+\frac{4}{9} f_{h}^{2}\right) \frac{\sqrt{1+\eta_{s}}}{1+\lambda_{s}} \\
& \eta_{s^{\prime}} f^{\prime}=\frac{5}{2} m-2 f^{\prime}+\frac{10}{21} m^{2}+\frac{4}{7} f_{h}^{2}-\frac{6}{7} m f_{h}
\end{aligned}
$$

The second order terms in Eq. (29), i.e., the terms containing $f_{h}^{2}$ and $m f_{h}$, ultimately become $0\left(f^{3}\right)$ when they are multiplied by $f$ on substitution in Eq. (30) and drop out. Their inclusion is desirable, however, to show exactly where the different terms become negligible. With the exception of the second-order terms, Eq. (29) and (30) are the same as de Sitter's equations (21) and (22) in his 1924 paper. 
The method adopted by de Sitter is discussed in detail in numerous papers (de Sitter, 1924, 1938; Bullard, 1948; Khan, 1968a). H is taken as the initial datum and an attempt is made to find a value of $J_{h}$ which would be compatible with the selected value of $H$ (both $J_{h}$ and $H$ being functions of $\mathrm{m}$ ). This is done by estimating a quantity $\mathrm{q}=3 / 2 \mathrm{C} / \mathrm{Ma}_{\mathrm{e}}{ }^{2}$ $\left(\mathrm{J}_{\mathrm{h}}=\mathrm{qH}\right)$ from a knowledge of the internal density distribution of the earth (de Sitter, 1924; Bu1lard, 1948). Once $J_{h}$ is determined, Eq. (5) will give the corresponding hydrostatic flattening. A serious disadvantage of the solution obtained by this method is that the moment of inertia of the hydrostatic model, constructed in this way, is greater than that of the real earth. This creates some dynamical complications as discussed before. However, it must be appreciated that de Sitter's whole effort was really directed to devise a method which would give the best approximation to the real flattening of the earth, not necessarily the hydrostatic flattening, in the context of the information available in his time.

Other post-satellite methods:

Jeffreys (1963) has given an excellent numerical method based on a simplified density model. He computes the various hydrostatic parameters from the first-order theory and evaluates the second-order correction terms by the numerical evaluation of the appropriate integrals. The method reported in this paper could be really regarded as a counterpart of Jeffreys' (1963) method with the exception that I have employed de Sitter's development of the hydrostatic theory.

In the previous applications of de Sitter's development to compute the hydrostatic flattening, however, it is usually claimed that using satellite-determined $\mathrm{J}$ and dynamical flattening $\mathrm{H}$, and hence knowing the polar moment of inertia of the earth, $f_{h}$ should be computed from the hydrostatic equations alone without using any controls from the external potential theory. If this is accepted, the use of de Sitter's (1924) equations is automatically ruled out because these equations are derived with the help of external potential theory. Consequent1y, the modified equations (Khan, 1968a) should be used, but in that event, the equations cannot be solved because of the explicit 
TABLE 3. Comparison of Hydrostatic Flattening Values*

\section{Pre-Sate111te Method}

(Based on the dynamfcal flattening $H$ as the inftial datum)

\begin{tabular}{lll} 
& & \multicolumn{1}{c}{$f_{h}^{-1}$} \\
de Sitter & $(1924)$ & $296.92 \pm 0.136$ \\
de Sitter & $(1938)$ & $296.753 \pm 0.086$ \\
Bullard & $(1948)$ & $297.338 \pm 0.050$ \\
Jeffreys & $(1952)$ & $297.299 \pm 0.071$ \\
Jeffreys & $(1963)+$ & $296.75 \pm 0.05$
\end{tabular}

Resulto Obtained After the Satelite-determination of $\mathrm{J}$

$f_{h}=$ hydrostatic flattentng; $f=1 / 298.25 \pm 0.05$

(a) Based on the polar moment of Inertia as the Initial datum

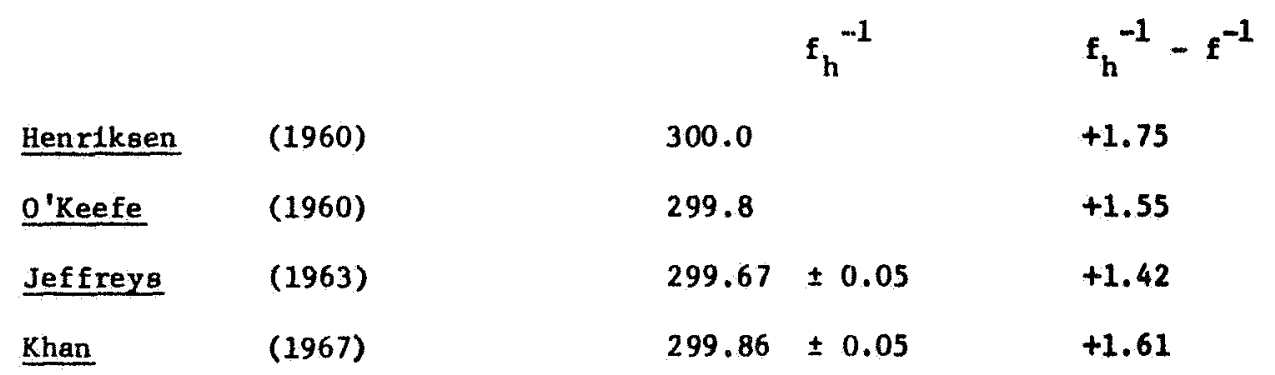

(b) From a solution of the modified equations alone, using polar moment of inertia and the satellite-determined $J$

$$
\begin{array}{lll}
296.70 & \pm 0.05 * * & -1.55 \\
297.04 & \pm 0.05 t t & -1.21
\end{array}
$$

*See also Table 1.

tUaing the pre-satelite approach.

**Based on $\mathrm{m}=0.00344980$ (Khan, 1967a; Jeffreys, 1963), H $=0.00327364$ (Khan, 1967), and $\mathrm{J}_{2}=0.001082645(\mathrm{Koza1}, 1964)$.

t+Based on $m=0.00344992$ (Henriksen, 1960), $H=0,00327070$, and $\mathrm{J}_{2}=0.00108270$. 
appearance of $J_{h}$ on the right-hand side of the equations and because of the necessity of treating this quantity as an unknown in the solution. If these modified hydrostatic equations are solved with the help of satellite-determined $J$ (i.e., $J=J_{h}$ ) one will obtain the results shown in the last section of Table 2. The first part of Table 2 gives the results obtained from different investigators using de Sitter's equations in a way which is pertinent to the discussion given in this report.

Table 3 lists the results obtained from the modified hydrostatic equations (Khan, 1968a) along with the results of other investigators who obtained their values using de Sitter's equations.

The important work of Ledersteger (1967) is not discussed because the discussion given here does not pertain to his method.

\section{Summary and Conclusions}

Since the hydrostatic geopotential coefficient $J$, appearing explicitly on the right-hand side of the modified hydrostatic equations, must be treated as an unknown in the solution of the hydrostatic equilibrium problem, these equations become difficult to solve all by themselves and one has to look for an additional. boundary condition. This boundary condition is inherent in the definition of the hydrostatic equilibrium and is derived from the external potential theory which neither assumes nor discounts the existence of hydrostatic equilibrium in the earth's interior. It requires that the equilibrium figure of the earth coincide exactly with that predicted from the external potential theory, if hydrostatic equilibrium exists in the earth's interior, and is stated in terms of Eq. (5). The solution obtained with the help of this boundary condition turns out to be sufficiently general so that the three most frequently mentioned particular solutions in literature can be obtained from this by merely defining the appropriate initial datum. The only geophysically meaningful mode1, of course, remains the one in which the polar or mean moment of inertia is held equal to that of the real earth, calculated from the satellite-determined $J$ and the dynamical flattening $H$ computed via the constant of precession of the real earth. For 
this hydrostatic model the flattening is $\mathrm{f}_{\mathrm{h}}^{-1}=299.75$. The solution is significant in that it demonstrates the correct structure of the problem of hydrostatic equilibrium of the earth. Taking the equilibrium figure as reference, the minimum strength of the earth required to support the hydrostatic stresses (considering the inequality due to second zonal harmonic on ly) is $4.7 \times 10^{7}$ dynes $/ \mathrm{cm}^{2}$ in case the stresses are supported by strength down to the core, and $8.7 \times 10^{7}$ dynes $/ \mathrm{cm}^{2}$ In case the stresses are supported by strength down to a depth of 0.1 of the earth's radius.

The anomalous gravity field of the earth with respect to the equilibrium figure and the international reference ellipsoid is shown in Figures 4 and 5. The equilibrium figure provides the best reference for computing the anomalous field because such a field would also reflect the hydrostatic stresses which become very important in geophysical studies on a regional scale. A comparison of Figures 4 and 5 shows the extent to which the anomalous gravity field depends on the reference figure used. Figures 6 and 7 show geoidal unalations with respect to the international reference ellipsoid and the equilibrium figure. Figure 8 showe the astrogeoid referred to the equilibrium figure. The agreement between the various extremums in Figures 7 and 8 is noteworthy and reflects the appropriateness of the reference figure to which both geoids are referred.

\section{Acknow ledgments}

The work was supported in part by NASA Grant No. NGR 12-001-045. The author is obligated to Dr. George H. Sutton, Professor of Geophysics at the Hawaii Institute of Geophysics, Honolulu, Hawaii, for some critical and informative discussions. 
(Footnote for p. 17)

It was brought to the author's attention that this was also recognized by Caputo (1965), who compared the hydrostatic flattening obtained from several different assumptions. However, Caputo did not point out the problem mentioned in this report, 1.e., the incompleteness of the system of equations (15 and (16), if $J_{h}$ is treated as an unknown, and, hence, does not deal with the problem considered in this report. 
Bullard, E. C., The figure of the earth, M.N.R.A.S., Geophys. Suppl. 5, 186-192, 1948.

Bullen, K. E., The problem of the earth's density variation, Bull. Seism. Soc. Am., 30, 235-250, 1940 .

Bullen, K. E., The density distribution of the earth's central core, Bu11. Seism. Soc. Am., 32, 19-29, 1942.

Caputo, M., The minimum strength of the earth, J. Geophys. Res., 70, 955-963, 1965.

C1airaut, A. C., Theorie de la Figure de la Terre, Paris, 1743.

de Sitter, W., On the flattening and the constitution of the earth,

Bu11. Astr. Insts. Netherlands, 2, 55, 97-108, 1924.

de Sitter, W., On the system of astronomical constants, Bull. Astr.

Insts. Netherlands, 8, 307, 213-229, 1938.

Fischer, I., Deviations of the geoid from an equilibrium figure,

Army Map Service, Washington, D. C., 1967.

Gaposhkin, E. M., A dynamical solution for the tesseral harmonics of the geopotential and for station coordinates (abstract), Trans. Am. Geophys. Union, 47, 47, 1966.

Hayford, J.F., The figure of the earth and isostasy, U.S.C.\&.G.S., 1909 .

Hayford, J. F., Supplementary investigations in 1909, U.S.\& S.G.S., 1910 .

Helmert, F. R., S. B. Preuso. Akad. Wiss., 10-19, 1911.

Henriksen, S. W., The hydrostatic flattening of the earth, Ann. Int. Geophys. Year, 12, 197-198, 1960.

Jeffreys, H., The stress differences in the earth's she11, M.N.R.A.S., Geophys. Supp1. 5, 71-89, 1943.

Jeffreys, H., The figure of the earth and the moon, M.N.R.A.S., Geophys. Supp1. 5, 219-247, 1948.

Jeffreys, H., The Earth, Cambridge University Press, Cambridge, 1952, 1962 .

Jeffreys, H., On the hydrostatic theory of the figure of the earth, Geophys. J., 8, 196-202, 1963. 
Jones, H. S., Dimensions and rotation, Chapt. I, In G. P. Kuiper (ed.) The Earth as a Planet, The University of Chicago Press, Chicago, 1954.

Khan, M. A., Some parameters of a hydrostatic earth, Trans. Am. Geophys. Union, 48, 1, 56, 1967.

Khan, M. A., A re-evaluation of the theory for the hydrostatic figure of the earth, J. Geophys. Res., 73, 5335-5343, 1968a.

Khan, M. A., General solution of the problem of hydrostatic equilibrium of the earth, (in press), 1968b.

King-Hele, D. G., G. E. Cook, and J. M. Rees, Determination of the even harmonics in the earth's gravitational potential, Geophys. J., 8, $119,1963$.

Kozai, Y., New determination of zonal harmonic coefficients of the earth's gravitational potential, Smith. Astrophys. Obsy. Sp. Rept. No. $165,1964$.

Ledersteger, K., Critical notes on mean functions derived from orbital perturbations of artificial satellites, In G. Veis (ed.), The Use of Artificial Satellites for Geodesy, vol. II, National Technical University, Athens, Greece, 1967.

Munk, W. H. and G. J. F. MacDonald, Continentality and the gravitational field of the earth, J. Geophys. Res., 65, 7, 2169-2172, 1960.

o'Keefe, J. A., Determination of the earth's gravitational field, In H. Kallmann (ed.), Space Research I, 448-457, 1960.

0 'Keefe, J. A., Letter to the Symposium on the determination of the figure of the earth, Studia Geophysica et Geodaetica, 2, 9, 1965.

0 'Keefe, J. A. and W. M. Kaula, Stress differences and the reference ellipsoid, Science, 142, 3590, 1963.

Poincare, H., Bu11. Astronomique France, 27, 321-356, 1910.

Rabe, E., The precessional constant from Eros, Astronom. J., 55, 181, 1950. Radau, R., Compte Rendus De Science, C. R. Acad. Sci. Paris, 100, 972-974, 1885 .

Tisserand, F., Mecaniqué Celeste, 4 Vols., Paris, 1889-1896. 